DOI 10. 18307/2022. 0225

(C) 2022 by Journal of Lake Sciences

\title{
过坝下泄水流中温室气体排放速率的数值模拟
}

\author{
黄菊萍 ${ }^{1,2}$, 欧洋铭 ${ }^{3}$, 李 然 $^{2}$, 冯镜洁 ${ }^{2 *}$, 李 哲 $^{1}$ \\ (1: 中国科学院重庆绿色智能技术研究院,重庆 400714) \\ (2:四川大学水力学与山区河流开发保护国家重点实验室,成都 610065) \\ (3: 成都大学建筑与土木工程学院,成都 610106)
}

\begin{abstract}
摘 要: 当水流通过泄洪建筑物下泄时, 水体中所溶解的温室气体 (二氧化碳 $\left(\mathrm{CO}_{2}\right)$ 、甲烷 $\left(\mathrm{CH}_{4}\right)$ 等) 会因为所受压力的 瞬间改变而导致溶解度降低, 从而造成气液之间传质的发生及水中温室气体的排放. 然而, 目前对于泄流条件下水中温 室气体排放的研究还较为缺乏. 鉴于原型观测与模型试验的局限性, 本文建立了大坝泄流条件下温室气体排放速率的数 学模型, 模型基于 VOF ( volume of fluid)气液两相流模型, 考虑了温室气体在过坝下泄过程中发生的气泡传质和自由液面 传质. 本文以温室气体 $\mathrm{CO}_{2}$ 和 $\mathrm{CH}_{4}$ 为研究对象, 分别通过模拟溶解态 $\mathrm{CO}_{2}$ 和 $\mathrm{CH}_{4}$ 在过流坝面和空中挑射过程中及在坝下 消力池或水垫塘内的输移扩散,计算得到 $\mathrm{CO}_{2}$ 和 $\mathrm{CH}_{4}$ 在水中的浓度分布及在不同上游来流的温室气体浓度工况下的坝下 温室气体排放速率. 模拟结果表明, 坝下温室气体在水中的浓度分布主要受到上游来流浓度大小、气液传质的发生及溶 解气体输移扩散的影响. 其中, 上游来流的温室气体浓度大小为影响坝下温室气体排放速率的主要因素. 本研究为明确 不同泄洪方式下的温室气体排放速率的大小和科学评估水电碳足迹提供了新的研究思路和技术基础.
\end{abstract}

关键词: 温室气体; 过坝下泄; 数值模拟; 排放速率

\section{Greenhouse gas emission rate under discharge condition by numerical simulation*}

\author{
Huang Juping ${ }^{1,2}$, Ou Yangming ${ }^{3}$, Li Ran $^{2}$, Feng Jingjie ${ }^{2 * *} \&$ Li Zhe ${ }^{1}$ \\ ( 1: Chongqing Institute of Green and Intelligence Technology, Chinese Academy of Sciences, Chongqing 400714, P.R.China) \\ (2: State Key Laboratory of Hydraulics and Mountain River Engineering, Sichuan University, Chengdu 610065, P.R. China) \\ (3: College of Architecture and Civil Engineering, Chengdu University, Chengdu 610106, P.R.China)
}

Abstract: The solubility of dissolved greenhouse gas ( $\mathrm{GHG})$, such as carbon dioxide $\left(\mathrm{CO}_{2}\right)$ and methane $\left(\mathrm{CH}_{4}\right)$ in water will be decreased under discharge conditions due to the sudden change of pressure, which will lead to gas-liquid mass transfer and GHG emission. However, the research about GHG emission under flood discharge conditions is still rare. Considering the limitation of prototype observation and model experiment, a mathematical model for GHG emission rate under flood discharge conditions was built in this paper. The model is based on the VOF (volume of fluid) method and takes into account the bubble mass transfer and free surface mass transfer. We simulated the transport and diffusion of dissolved $\mathrm{CO}_{2}$ and $\mathrm{CH}_{4}$ in the spillway and jet flow respectively, and calculated their concentration distribution and emission rate. The simulated results show that the concentration distribution of GHG downstream of the dam is mainly affected by the upstream inflow concentration, gas-liquid mass transfer, the transport and diffusion of dissolved GHG. Among them, the concentration of GHG in the upstream flow is the main factor affecting the emission rate downstream of the dam. This study provides a new research approach and technical base for clarifying the amount of GHG emission rate under different discharge types, and scientifically evaluating the hydroelectric carbon footprint.

Keywords: Greenhouse gas; discharge through dam; numerical simulation; emission rate

温室气体主要包括二氧化碳 $\left(\mathrm{CO}_{2}\right)$ 、甲烷 $\left(\mathrm{CH}_{4}\right)$ 和氧化亚氮 $\left(\mathrm{N}_{2} \mathrm{O}\right)$ 等 ${ }^{[1]}$. 化石燃料能源的利用、森林的

* 2021-06-05 收稿;2021-08-18 收修改稿.

国家自然科学基金项目 (52039006)、国家自然科学基金国际合作项目 (51861125204) 和四川省科技计划项目 (2018JY0598) 联合资助.

** 通信作者; E-mail: fengjingjie@ scu.edu.cn. 
砍伐、人工湿地的形成和畜牧业的大规模发展等人类活动会显著增加大气中的温室气体浓度,引起地球平 均温度的升高从而导致全球气候变暖, 对人类生产和生活带来了巨大的负面影响 ${ }^{[2-4]}$. 水力发电将天然水体 的势能和动能转化为电能输出, 可大幅减少 $\mathrm{CO}_{2} 、 \mathrm{CH}_{4}$ 等温室气体的排放量, 是迄今唯一技术已发展成熟、可 大规模开发的再生能源. 然而, 筑坝蓄水将不可避免地淹没一定数量的土地, 改变水文情势, 迫使原有区域 生态系统同大气之间温室气体的源汇关系发生变化 ${ }^{[5]}$. 水库温室气体源汇变化是近年来水利水电工程开发 和运行的重要关注问题之一.

目前, 水库温室气体排放的主要途径有: 1) 水库库区与下游受影响河道水一气界面扩散释放;2) 近岸浅 水区域可能出现的气泡释放; 3) 库岸带或水库消落带的温室气体排放; 4) 水体流经大坝 (包括泄流建筑物及 水轮机组等) 时, 因压力和温度的瞬间改变, 导致温室气体产生消气释放 (degassing). 不同的水库在不同季 节和运行方式下的消气释放通量存在明显的时空异质性 ${ }^{[6]}$, 其对水库温室气体总排放量的贡献亦有显著差 别. 已有研究发现, 水库 $\mathrm{CO}_{2}$ 的消气释放量可占到其运行期间 $\mathrm{CO}_{2}$ 总排放量的 $3.28 \% \sim 57.14 \%$; 而 $\mathrm{CH}_{4}$ 的消 气释放量可占到水库 $\mathrm{CH}_{4}$ 总排放量的 $2.05 \% \sim 51.89 \%^{[7-10]}$. 尽管如此, 当前全球水库温室气体消气释放的研 究仍十分有限. 另一方面, 当前水库温室气体消气排放估算中, 多将水库视为一个整体, 在临近大坝的上下 游设置控制断面, 结合两个控制断面间的浓度差和流量变化进行估算. 由于温室气体在过坝下泄过程 (泄 洪) 与在发电设施中的释放机理并不相同, 当前的水库温室气体消气释放研究, 鲜有对这两种消气释放方式 分别予以考虑.

本研究拟针对溶解态温室气体在过坝下泄中的传质和输运过程进行数值模拟分析, 以大渡河铜街子电 站和瀑布沟电站为例, 针对典型泄洪工况, 模拟计算温室气体在溢流坝面和空中挑射过程中及坝下水垫塘 或消力池内的浓度变化及排放速率, 为明确不同泄洪方式下的温室气体消气释放强度、准确核算水库不同 服务功能下温室气体排放速率提供新的研究思路和方法基础.

\section{1 计算模型的建立}

目前国内外尚未有关于温室气体过坝下泄数值模拟研究的报道, 而四川大学、清华大学及美国爱荷华 大学等机构对总溶解气体和溶解氧的过坝下泄过程进行了卓有成效的数值模拟研究 ${ }^{[11-13]}$. 本研究基于 Yang 等 ${ }^{[14]}$ 建立的关于总溶解气体的立面二维气液两相流模型, 在已有模型的基础上, 考虑 $\mathrm{CO}_{2} 、 \mathrm{CH}_{4}$ 等温室气体 的理化性质,增加了温室气体排放速率计算公式,建立了温室气体过坝下泄排放速率计算模型.

\section{1 模型结构}

Yang 等 ${ }^{[14]}$ 提出气体在水气界面的传质可分为气泡界面传质和水气自由表面传质. 水坝泄水时, 水流在 溢流坝面或空中挑射的过程中, 由于压力的瞬间改变, 水体中温室气体的溶解度降低, 部分温室气体将以气 泡的形式从水中析出; 在坝下, 大量的空气被泄洪水流卷吸进人水中, 并在水中形成大量气泡, 当气泡流至 坝下消力池或水垫塘底部时, 由于消力池和水垫塘内气体的承压急剧增大, 气体溶解度较常压下显著增加, 导致温室气体通过气泡传质从气泡溶解进人水中. 此外, 在自由液面还存在溶解态温室气体与大气之间的 传质发生过程, 温室气体过坝下泄的传质过程如图 1 所示.

\section{2 水动力学方程}

温室气体排放速率计算模型通过 VOF ( volume of fluid) 气液两相流模型计算自由液面, 采用 RANS (Reynolds-averaged Navier-Stokes) 紊动模型进行求解.

模型的连续性方程和动量方程由以下公式表示:

$$
\begin{gathered}
\nabla \cdot \boldsymbol{U}=0 \\
\frac{\partial(\rho \cdot U)}{\partial t}+\nabla \cdot(\rho \cdot \boldsymbol{U} \cdot \boldsymbol{U})=-\nabla P+\nabla \cdot(\mu \cdot \nabla \boldsymbol{U})+\rho \cdot f_{\mathrm{b}} \\
\frac{\partial \alpha}{\partial t}+\nabla \cdot(\boldsymbol{U} \cdot \alpha)=0 \\
\rho=\rho_{\mathrm{w}} \cdot \alpha_{\mathrm{w}}+\rho_{\mathrm{g}}\left(1-\alpha_{\mathrm{w}}\right) \\
\mu=\mu_{\mathrm{w}} \cdot \alpha_{\mathrm{w}}+\mu_{\mathrm{g}}\left(1-\alpha_{\mathrm{w}}\right)
\end{gathered}
$$




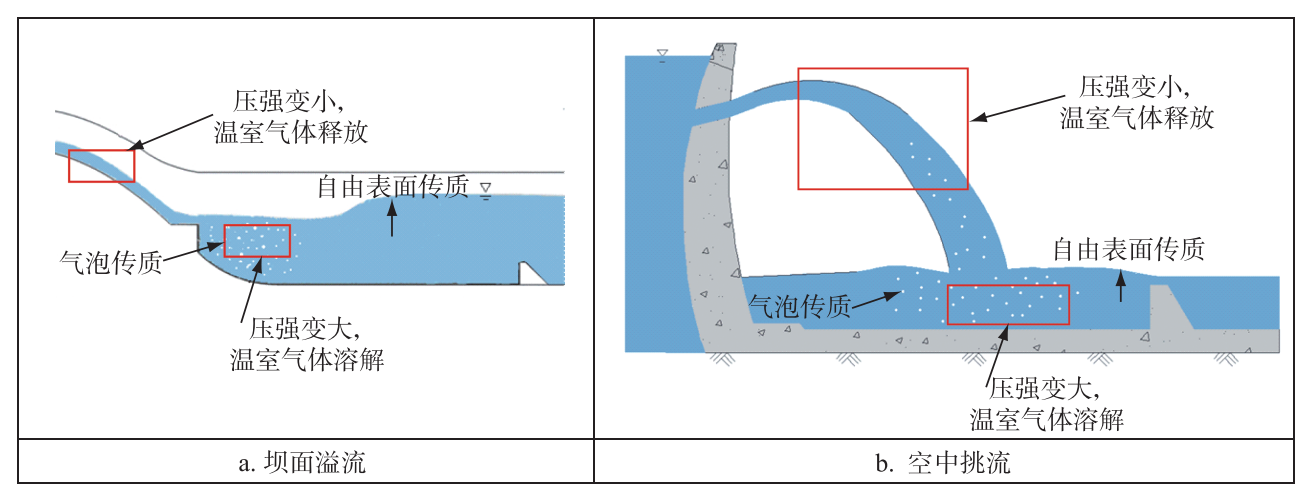

图 1 温室气体过坝下泄传质过程示意图

Fig.1 Mass transfer process of GHG with discharge

式中, $\rho$ 为水气混合密度 $\left(\mathrm{kg} / \mathrm{m}^{3}\right)$, 根据水气两相体积分数加权计算得到, 见式 $(4) ; \mu$ 为动力粘度 $(\mathrm{kg} /(\mathrm{m}$. $\mathrm{s})) ; f_{\mathrm{b}}$ 为考虑了重力和表面张力的相间表面上的单位质量上的体积力 $\left(\mathrm{m} / \mathrm{s}^{2}\right), P$ 为压强 $(\mathrm{Pa})$. $\alpha$ 为体积分 数;下标 $\mathrm{w}$ 表示水相;下标 $\mathrm{g}$ 表示气相.

模型采用标准 $k-\varepsilon$ 紊流模型, 相关方程如下:

$$
\begin{gathered}
\frac{\partial(\rho k)}{\partial t}+\nabla \cdot(\rho k U)=\frac{\partial}{\partial x_{j}}\left[\left(\mu+\frac{\mu_{\mathrm{t}}}{\sigma_{k}}\right) \frac{\partial k}{\partial x_{j}}\right]+G_{k}-\rho \varepsilon \\
\frac{\partial(\rho \varepsilon)}{\partial t}+\nabla \cdot(\rho \varepsilon U)=\frac{\partial}{\partial x_{j}}\left[\left(\mu+\frac{\mu_{t}}{\sigma_{\varepsilon}}\right) \frac{\partial \varepsilon}{\partial x_{j}}\right]+\frac{C_{1 \varepsilon} \varepsilon}{k} G_{k}-C_{2 \varepsilon} \rho \frac{\varepsilon^{2}}{k}
\end{gathered}
$$

式中, $k$ 为紊动动能 $\left(\mathrm{m}^{2} / \mathrm{s}^{2}\right) ; \varepsilon$ 为紊动动能耗散率 $\left(\mathrm{m}^{2} / \mathrm{s}^{3}\right) ; \mu_{\mathrm{t}}$ 为育流粘性系数 $(\mathrm{kg} /(\mathrm{m} \cdot \mathrm{s})) ; G_{k}$ 为由平均速 度梯度引起的紊动动能产生项 $\left(\mathrm{kg} /\left(\mathrm{m} \cdot \mathrm{s}^{3}\right)\right) ; \sigma_{k}$ 为紊动能所对应的 Prandtl 数; $\sigma_{\varepsilon}$ 为紊动能耗散率所对应的 Prandtl 数; $C_{1 \varepsilon} 、 C_{2 \varepsilon}$ 分别为紊流模型计算参数.

\section{3 温室气体输移扩散方程}

因过坝下泄消气释放过程时间短, 忽略温室气体在水体中发生的电化反应和可能存在的生物转化, 通 用的温室气体浓度输移扩散方程为:

$$
\frac{\partial C}{\partial t}+\nabla \cdot(\boldsymbol{U} C)=\nabla \cdot\left[\left(\nu_{\mathrm{m}}+\frac{\nu_{\mathrm{t}}}{S c}\right) \nabla C\right]+S_{\mathrm{b}}+S_{\mathrm{s}}
$$

式中, $C$ 为某种温室气体在水中的浓度 $\left(\mathrm{mol} / \mathrm{m}^{3}\right) ; S c$ 为 Schmidt 数; $\nu_{\mathrm{t}}$ 为紊动扩散系数 $\left(\mathrm{m}^{2} / \mathrm{s}\right) ; \nu_{\mathrm{m}}$ 为分子扩散 系数 $\left(\mathrm{m}^{2} / \mathrm{s}\right) ; S_{\mathrm{b}}$ 为气泡溶解于水中或从水中析出的温室气体浓度源项 $\left(\mathrm{mol} /\left(\mathrm{m}^{3} \cdot \mathrm{s}\right)\right) ; S_{\mathrm{s}}$ 为水气自由界面传 质源项 $\left(\mathrm{mol} /\left(\mathrm{m}^{3} \cdot \mathrm{s}\right)\right)$.

1.3.1 气泡传质源项 目前已有相关研究对气泡的界面传质进行了分析 ${ }^{[15-16]}$, 本研究采用的气泡传质源项 计算公式如下:

$$
S_{\mathrm{b}}=f \frac{6 \alpha_{\mathrm{g}} \cdot k_{\mathrm{b}}}{D_{\mathrm{b}}}\left[C_{\mathrm{atm}}\left(1+\frac{P}{P_{\mathrm{atm}}}\right)-C\right]
$$

式中, $C_{\mathrm{atm}}$ 为在对应当地大气压和当时温度条件下该气体的饱和溶解度 $\left(\mathrm{mol} / \mathrm{m}^{3}\right) ; P 、 P_{\mathrm{atm}}$ 分别为压强和标准大 气压 $(\mathrm{Pa}) ; D_{\mathrm{b}}$ 为气泡粒径 $(\mathrm{m}) ; f$ 为某种温室气体在空气中的组分比例; $k_{\mathrm{b}}$ 为紊动气泡界面传质系数 $(\mathrm{m} / \mathrm{s})$.

$$
\begin{gathered}
k_{\mathrm{b}}=\beta \frac{2 \nu_{\mathrm{m}} \cdot P e_{\mathrm{b}}^{1 / 2}}{\sqrt{\pi} D_{\mathrm{b}}}\left(1-\frac{2}{3\left(1+0.09 R e_{\mathrm{b}}^{2 / 3}\right)^{3 / 4}}\right) \\
\operatorname{Re}_{\mathrm{b}}=\frac{\rho_{\mathrm{w}} \cdot D_{\mathrm{b}}\left|\boldsymbol{U}_{\mathrm{br}}\right|}{\mu_{\mathrm{w}}} \\
P e_{\mathrm{b}}=D_{\mathrm{b}}\left|\boldsymbol{U}_{\mathrm{br}}\right| / \nu_{\mathrm{m}}
\end{gathered}
$$


式中, $\beta$ 为模型参数, 需通过率定得到; $R e_{\mathrm{b}}$ 为气泡雷诺数; $P e_{\mathrm{b}}$ 为气泡 Peclet 数; $\boldsymbol{U}_{\mathrm{br}}$ 为气泡相对运动速度 $(\mathrm{m} /$ $\mathrm{s}) ; \mu_{\mathrm{w}}$ 为水相动力粘度 $(\mathrm{kg} /(\mathrm{m} \cdot \mathrm{s}))$.

1.3.2 自由表面传质源项

$$
S_{\mathrm{s}}=\frac{k_{1}}{\Delta Z}\left(C_{\mathrm{atm}}-C\right)
$$

式中, $\Delta Z$ 为表层网格高度 $(\mathrm{m}) ; k_{1}$ 为自由表面传质系数 $(\mathrm{m} / \mathrm{s})^{[17]}$.

$$
k_{1}=k_{600}\left(\frac{S c_{\mathrm{b}}}{600}\right)^{-x}
$$

式中, $x$ 为常数, 取值受风速影响; $k_{600}$ 是在 $20^{\circ} \mathrm{C}$ 时 $S c h$ midt 常数等于 600 的气体传输速度 $(\mathrm{m} / \mathrm{s}) ; S c_{\mathrm{b}}$ 为温室 气体的 Schmidt 常数, 取决于温度高低.

对于 $\mathrm{CO}_{2}$ 和 $\mathrm{CH}_{4}$ 有:

$$
\begin{gathered}
S c_{\mathrm{b}}\left(\mathrm{CO}_{2}\right)=1911.1-118.11 t+3.4527 t^{2}-0.04132 t^{3} \\
S c_{\mathrm{b}}\left(\mathrm{CH}_{4}\right)=1897.8-114.28 t+3.2902 t^{2}-0.039061 t^{3}
\end{gathered}
$$

式中, $t$ 为温度 $\left({ }^{\circ} \mathrm{C}\right)$, 本研究采用温室 $20^{\circ} \mathrm{C}$ 进行计算.

\section{4 温室气体排放速率方程}

$$
E=Q \cdot \Delta C
$$

式中, $E$ 为温室气体向大气中的排放速率 $(\mathrm{mol} / \mathrm{s}) ; Q$ 为水流流量 $\left(\mathrm{m}^{3} / \mathrm{s}\right) ; \Delta C$ 为上下游断面温室气体浓度差 $\left(\mathrm{mol} / \mathrm{m}^{3}\right)$.

\section{5 数值求解}

本研究基于 OpenFOAM 开源平台开展模拟求解, 以 interFoam 求解器为基础, 在计算流场、压力场的基 础上添加温室气体浓度输运方程, 以 PIMPLE 算法反映压力变化对速度场的影响. PIMPLE 算法将 SIMPLE ( Semi-Implicit Method for Pressure-Linked Equations)算法和 PISO (Pressure Implicit with Splitting of Operators) 算法结合起来, PIMPLE 的基本思想是在每个时间步长内用 SIMPLE 稳态算法求解, 时间步长的步进用 PISO 算法来完成. 在方程离散格式上,瞬时项采用 Euler 格式; 对流项采用 Gauss linear 格式; 拉普拉斯项使用 Gauss linear corrected 格式. 具体信息详见 https://openfoam.org.

\section{2 温室气体过坝下泄模拟}

本研究分别考虑了坝面溢流和空中挑流 2 种情况. 在坝面溢流过程中, 由于水流发生自掺气而形成掺 气水流. 掺气水流增大了气液接触面积, 同时也改变了流场和压力分布, 从而促进了气液传质. 而空中挑流 则是将水流射向空中形成挑流状态, 水流完全暴露在大气中从而发生掺气. 在空中挑流情况下, 气液传质受 到水流破碎程度的影响.

\section{1 坝面溢流温室气体浓度分布: 以铜街子电站泄流工况为例}

采用大渡河铜街子水电站作为典型坝面溢流研究对象. 铜街子水电站位于大渡河梯级开发的下游河段, 如图 2 所示. 大坝为混凝土重力坝, 最大坝高 $82 \mathrm{~m}$, 总库容 2.0 亿 $\mathrm{m}^{3}$, 调节 库容 0.3 亿 $\mathrm{m}^{3}$, 为日、周调节水库. 溢流坝段全长 $105 \mathrm{~m}$, 分 5 个等宽坝块, 每个坝块设以开敞式溢流表孔. 铜街子工程泄水 采用底流消能形式, 在桩号 (坝) $0+160.0 \mathrm{~m}$ 处设置消力坎, 并 在护坦末端设置差动式尾坎, 起到二次消能的作用.

2.1.1 模拟工况设置四川大学于 2009 年对铜街子电站的坝 下过饱和总溶解气体进行了原型观测, 采用原型观测工况, 即 铜街子水电站 3\#溢流表孔泄水时作为计算工况, 对应泄水流 量为 $629 \mathrm{~m}^{3} / \mathrm{s}$, 上下游水位差为 $33.65 \mathrm{~m}$. 根据以往针对长江上 游梯级水库的温室气体浓度的实测资料可知, 长江上游梯级库

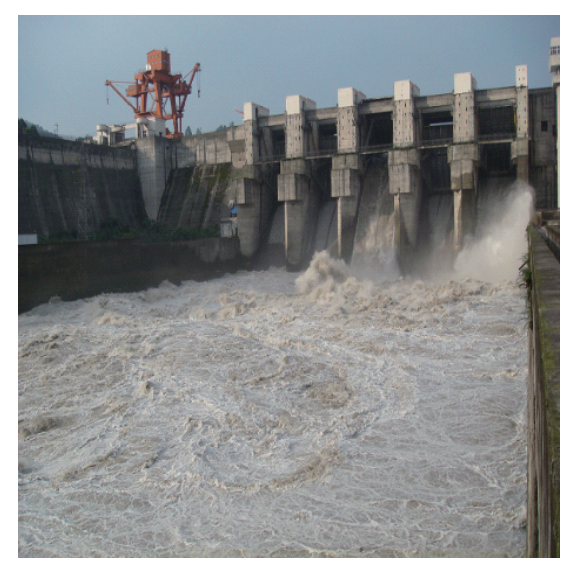

图 2 铜街子水电站泄水照片

Fig.2 The picture of Tongjiezi Dam 
区中的溶解态 $\mathrm{CO}_{2} 、 \mathrm{CH}_{4}$ 基本处于过饱和状态, 因此, 本研究设计了 4 组计算工况, 如表 1 所示, $\mathrm{CO}_{2} 、 \mathrm{CH}_{4}$ 的 上游来流饱和度范围分别为 $100 \% \sim 180 \%$, 各工况下的 $\mathrm{CO}_{2}$ 和 $\mathrm{CH}_{4}$ 的初始饱和度均为 $100 \%$. 为便于对比分 析,本研究引人了气体饱和度,其计算公式为:

$$
G=\frac{C}{C_{\text {atm }}} \times 100 \%
$$

式中, $G$ 为温室气体的饱和度 $(\%)$.

表 1 铜街子/瀑布沟电站计算工况设置

Tab.1 Settings of calculate working conditions of Tongjiezi/Pubugou Dam cases

\begin{tabular}{ccccc}
\hline \multirow{2}{*}{ 温室气体 } & \multicolumn{4}{c}{ 工况 } \\
\cline { 2 - 5 } & $\mathrm{tjz}-1 / \mathrm{pbg}-1$ & $\mathrm{tjz}-2 / \mathrm{pbg}-2$ & $\mathrm{tjz}-3 / \mathrm{pbg}-3$ & $\mathrm{tjz}-4 / \mathrm{pbg}-4$ \\
\hline $\mathrm{CO}_{2}$ 人流饱和度 $\%$ & 100 & 120 & 150 & 180 \\
$\mathrm{CH}_{4}$ 人流饱和度 $/ \%$ & 100 & 120 & 150 & 180 \\
\hline
\end{tabular}

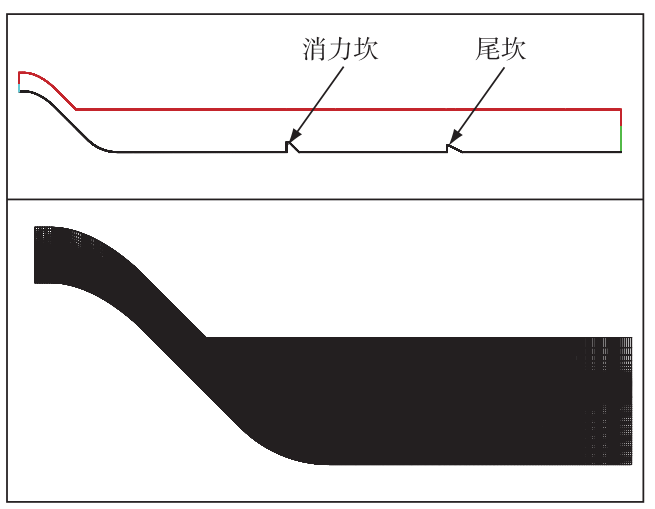

图 3 铜街子电站计算边界及网格划分示意图 (红色:空气人流;绿色:出流; 蓝色:速度人流;黑色:固壁)

Fig. 3 The boundary condition and mesh of Tongjiezi Dam cases

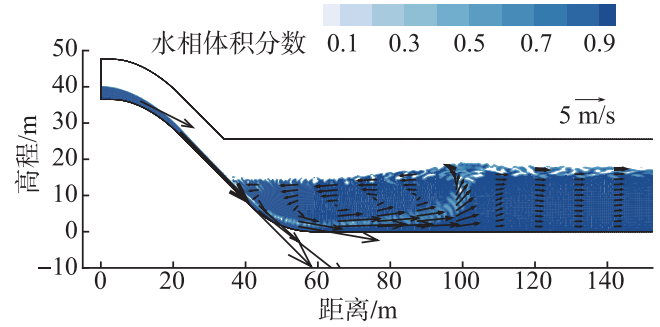

图 4 铜街子电站自由液面及速度矢量示意

Fig.4 The free surface and velocity vector of Tongjiezi Dam cases
2.1.2 网格划分及边界条件 计算区域纵向范围从铜 街子坝前到坝下约 $360 \mathrm{~m}$. 计算区域采用 2D 结构化 网格. 鉴于掺气浓度对网格尺寸要求较高, 在进行网 格划分时,着重对水气交界面进行加密处理. 该区域 网格密度较大, 网格尺寸大小仅 $0.2 \mathrm{~m}$ 左右, 在其他 区域纵向和垂向上,网格尺寸大小在 $0.5 \sim 1.5 \mathrm{~m}$ 之间. 铜街子电站计算区域网格划分及边界设置示意图见 图 3.

2.1.3 模型验证及参数取值 Yang 等 ${ }^{[14]}$ 根据原型观 测结果分别对铜街子电站的坝下总溶解气体饱和度 分布进行模型参数率定和验证. 结果表明, 当气泡粒 径均值取 $0.005 \mathrm{~m}$, 模型参数 $\beta$ 为 3.0 时, 铜街子电站 的坝下总溶解气体饱和度的计算值与实测值的误差 在 5\% 以内. 模拟结果表明, 在此模型参数取值情况 下, 该模型可以很好地模拟出过坝下泄过程中的气液 传质过程和溶解气体浓度分布.

2.1.4 模拟结果分析 图 4 为铜街子电站计算工况泄 水时水相体积分数分布及速度矢量图. 可以看出, 下 泄水流进人到消力池内造成了大量气泡卷吸人水体 中, 导致消力池内掺气浓度升高. 从流速矢量图可以看 出, 水流从溢流坝下泄至消力池时, 下泄水流与消力池 中的水体发生强烈碰撞, 消力池内流速急剧增大, 其中 高流速的主流在消力池底部, 最大流速达 $19 \mathrm{~m} / \mathrm{s}$. 在水 体潜人消力池底部过程中, 由于受到水体阻力, 小部分 水体向上冲击形成较大漩涡, 大部分主流水体流向下 游, 通过水跃产生表面旋滚和强烈紊动, 起到卷吸空气 进人水体并消能的作用.

图 5 为铜街子电站各计算工况的 $\mathrm{CO}_{2}$ 和 $\mathrm{CH}_{4}$ 的过 坝下泄饱和度模拟结果图. 从图中可以看出, 当高饱和 度的 $\mathrm{CO}_{2}$ 和 $\mathrm{CH}_{4}$ 随水流流经溢流坝面时, 受到压强的影响, $\mathrm{CO}_{2}$ 和 $\mathrm{CH}_{4}$ 的饱和度迅速降低. 当溶解态温室气 

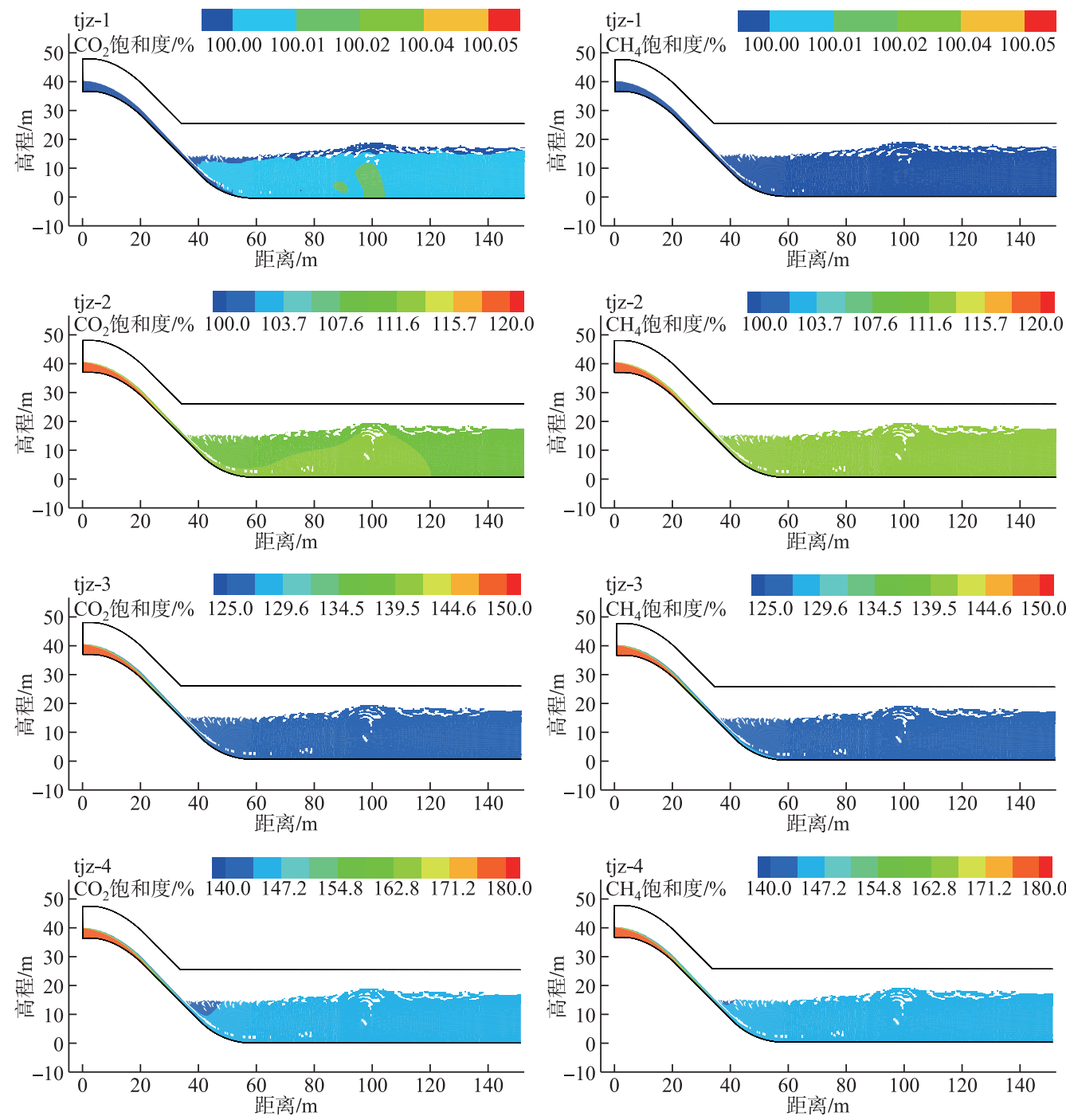

图 5 铜街子电站各工况温室气体过坝下泄模拟结果

Fig.5 The simulated results of GHG distribution with discharge under different working conditions of Tongjiezi Dam cases

体随流输移至坝下消力池内时, $\mathrm{CO}_{2}$ 和 $\mathrm{CH}_{4}$ 的饱和度沿程变化较小. 受到气液传质的影响, 温室气体的人流 饱和度越高, 其在溢流坝面上的释放量也越大. 对消力池出口断面处的 $\mathrm{CO}_{2}$ 和 $\mathrm{CH}_{4}$ 的饱和度进行分析, 结果 如图 6 所示,计算结果表明, 当温室气体人流浓度越高, 其在坝下的浓度变化值也越大, 但受到来流浓度的 影响, 坝下温室气体浓度也随人流浓度值变高. 从坝面溢流的模拟结果可以看出, 由于大气中的温室气体含 量比例较低, 坝下发生的气液传质程度受到限制, 因此上游来流的温室气体浓度大小为影响坝下温室气体 浓度分布的主要因素.

\section{2 空中挑流温室气体浓度分布: 以瀑布沟电站泄流工况为例}

采用大渡河瀑布沟水电站作为典型挑流研究对象, 如图 7 所示. 电站为堤坝式开发,坝址位于尼日河汇 口上游约 $700 \mathrm{~m}$ 的大渡河干流, 拦河建筑物为直心墙土石坝, 最大坝高 $186 \mathrm{~m}$. 电站装机容量为 $3300 \mathrm{MW}$, 多 年平均年发电量 145.8 亿 $\mathrm{kW} \cdot \mathrm{h}$, 水库正常蓄水位 $850 \mathrm{~m}$ 时, 总库容 50.64 亿 $\mathrm{m}^{3}$, 水库面积 $84.14 \mathrm{~km}^{2}$, 具有 

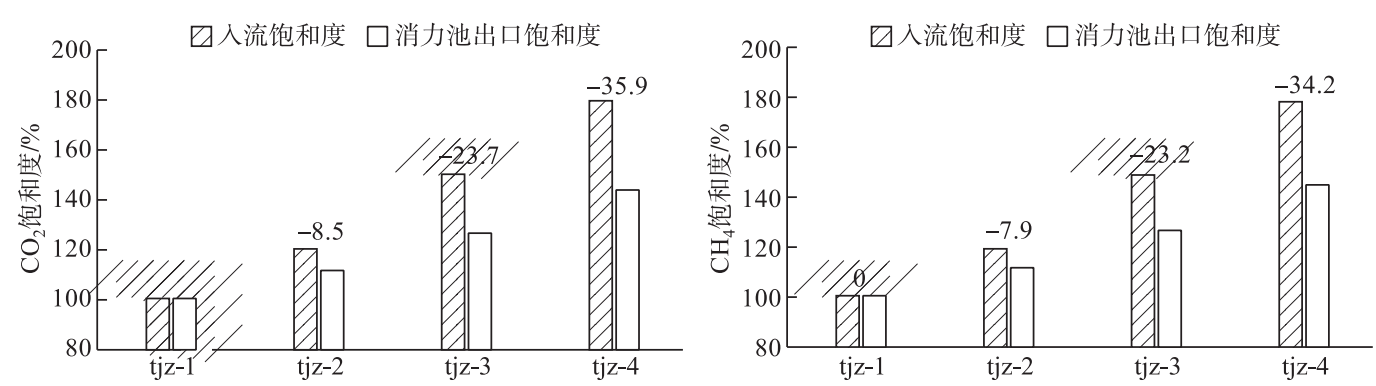

图 6 铜街子电站各工况温室气体消力池出口处饱和度结果 (图中数字为饱和度差值)

Fig. 6 The simulated results of GHG concentration at basin outlet under different working conditions of Tongjiezi Dam cases

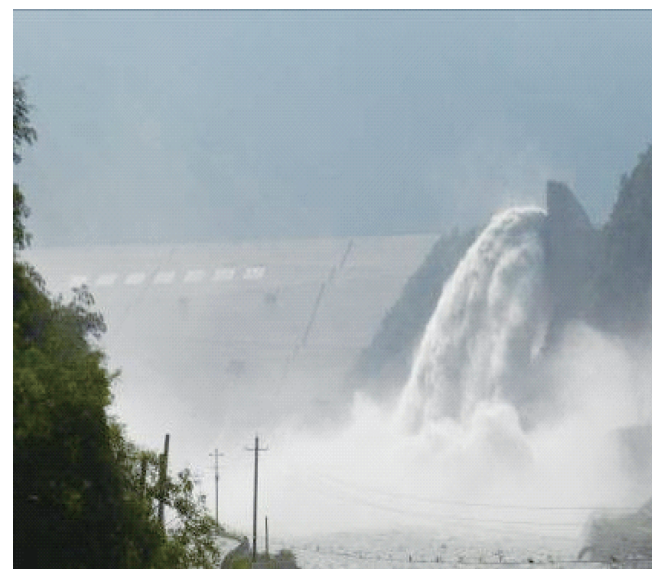

图 7 瀑布沟水电站泄水照片

Fig.7 The picture of Pubugou Dam

季调节性能. 瀑布沟水电站工程由大渡河主体工程与尼日河引水工程组成, 主体工程包括直心墙土石坝、左 岸岸边溢洪道、泄水隧洞、放空洞、左岸地下厂房; 尼日河引水工程包括泄水闸、进水口、挡水坝和引水隧洞. 其中, 左岸溢洪道总长 $578 \mathrm{~m}$, 由进口引渠段、进口溢流堰、泄槽段和出口段组成, 采用挑流消能方式. 深孔无 压泄水洞布置在左岸地下厂房左侧山体内, 采用无压隧洞, 出口位于厂房尾水出口下游, 采用挑流鼻坎消能. 2.2.1 模拟工况设置四川大学于 2009 年对瀑布沟电站的坝下过饱和总溶解气体进行了原型观测, 采用原 型观测工况, 即瀑布沟水电站溢洪道泄水时作为计算工况, 对应泄水流量为 $2570 \mathrm{~m}^{3} / \mathrm{s}$, 上下游水位差为 $170.5 \mathrm{~m}$. 本研究设计了 4 组计算工况, 如表 1 所示, $\mathrm{CO}_{2} 、 \mathrm{CH}_{4}$ 的上游来流饱和度范围分别为 $100 \% \sim 180 \%$, 各 工况下的 $\mathrm{CO}_{2}$ 和 $\mathrm{CH}_{4}$ 的初始饱和度均为 $100 \%$.

2.2.2 网格划分及边界条件 计算区域纵向范围从瀑布沟坝前到坝下约 $400 \mathrm{~m}$. 计算区域采用 $2 \mathrm{D}$ 结构化网 格. 鉴于掺气浓度对网格尺寸要求较高, 在进行网格划分时, 着重对水气交界面及冲坑区域进行加密处理. 该区域网格密度较大, 网格尺寸大小仅 $0.5 \mathrm{~m}$ 左右, 在其他区域纵向和垂向上, 网格尺寸大小在 $0.5 \sim 1.5 \mathrm{~m}$ 之间. 瀑布沟电站计算区域网格划分及边界设置示意图见图 8.

2.2.3 模型验证及参数取值 Yang 等 ${ }^{[14]}$ 根据原型观测结果分别对铜街子电站的坝下总溶解气体饱和度分 布进行了模型参数率定和验证. 研究结果表明, 当气泡粒径均值取 $0.005 \mathrm{~m}$, 模型参数 $\beta$ 为 1.0 时, 铜街子电 站的坝下总溶解气体饱和度的计算值与实测值的误差在 $5 \%$ 以内. 模拟结果表明, 在此模型参数取值情况 下,该模型可以很好地模拟出过坝下泄过程中的气液传质过程和溶解气体浓度分布. 


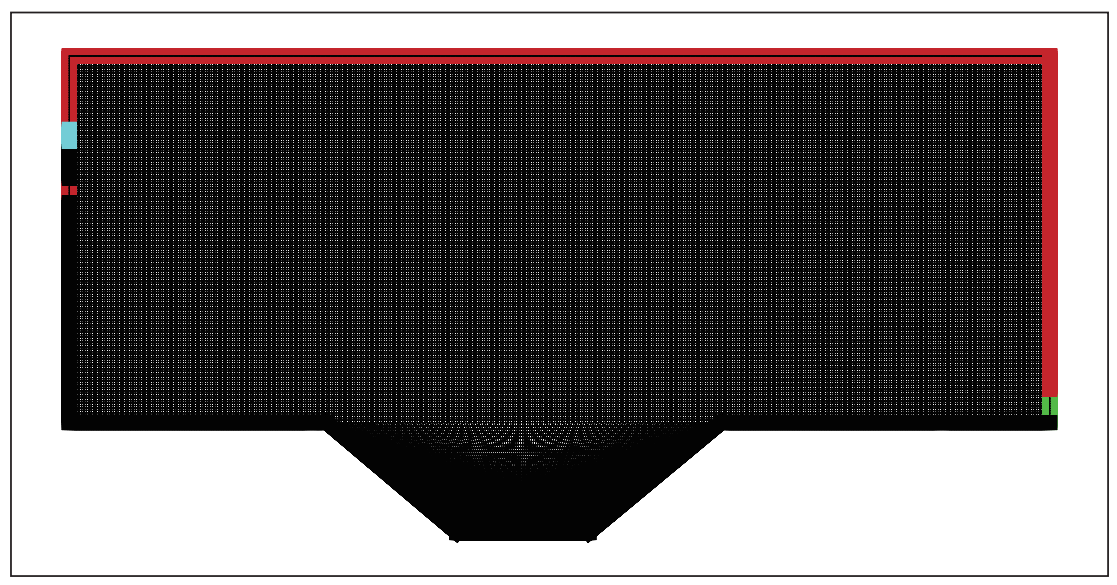

图 8 瀑布沟电站计算边界及网格划分示意图 (红色:空气人流; 绿色:出流; 蓝色: 速度人流; 黑色:固壁)

Fig. 8 The boundary condition and mesh of Pubugou Dam cases

\subsection{4 模拟结果分析 图 9 为瀑布沟电站计算工况} 泄水时水相体积分数分布及速度矢量图. 水流经溢 洪道挑坎末端挑射进人冲坑过程中, 挑射水流在空 中掺气现象明显, 大量空气被卷吸进人水体中. 虽然 冲坑底部距离挑坎出口高达 $160.8 \mathrm{~m}$, 挑射水流依然 能够进人水体深层, 直至冲坑底部, 造成水体深层掺 气剧烈. 从速度矢量分布可以看出, 水流挑射进人冲 坑过程中, 随着势能转化为动能, 水流速度不断增 加, 冲坑内最大流速高达 $45 \mathrm{~m} / \mathrm{s}$, 并在在水舌人水点 的上游及下游区域形成漩浴翻滚.

图 10 为瀑布沟电站各计算工况的 $\mathrm{CO}_{2}$ 和 $\mathrm{CH}_{4}$ 的 过坝下泄饱和度模拟结果图. 从图中可以看出, 当高

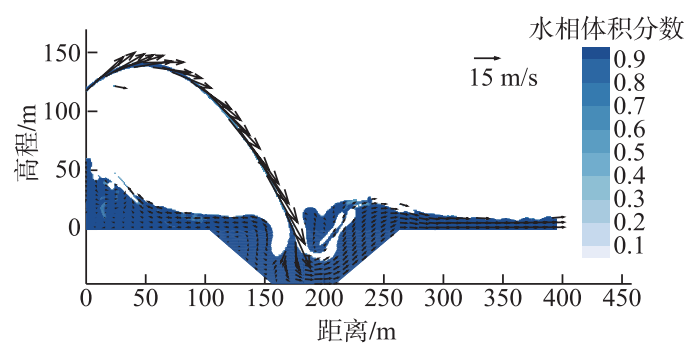

图 9 瀑布沟电站自由液面及速度矢量示意图

Fig.9 The free surface and velocity vector of Pubugou Dam cases

饱和度的 $\mathrm{CO}_{2}$ 和 $\mathrm{CH}_{4}$ 随水流经过空中挑射时, 受到压强的影响, $\mathrm{CO}_{2}$ 和 $\mathrm{CH}_{4}$ 的饱和度出现降低情况. 当溶解 态温室气体随流输移至坝下冲坑下游时, $\mathrm{CO}_{2}$ 和 $\mathrm{CH}_{4}$ 的饱和度沿程变化较小. 对坝下 $400 \mathrm{~m}$ 断面处的 $\mathrm{CO}_{2}$ 和 $\mathrm{CH}_{4}$ 的饱和度进行分析, 结果如图 11 所示. 计算结果表明, 当温室气体人流浓度越高, 坝下温室气体浓度也 越高. 从挑流的模拟结果可以看出, 由于大气中的温室气体含量比例较低, 坝下发生的气液传质程度受到限 制, 上游来流的温室气体浓度大小为影响坝下温室气体浓度分布的主要因素.

\section{3 温室气体排放速率计算结果分析}

根据式 (17), 可知温室气体排放速率由温室气体浓度差及水流流量决定. 分别取进口断面和铜街子电 站各计算工况下的消力池出口断面、瀑布沟电站各计算工况下的坝下 $400 \mathrm{~m}$ 断面为研究断面计算 $\mathrm{CO}_{2}$ 和 $\mathrm{CH}_{4}$ 的排放速率. 由于目前缺乏对大渡河库区温室气体浓度的监测数据, 参考 2018 年中科院重庆绿色智能 技术研究院针对三峡库区温室气体浓度的监测数据, 对于铜街子电站, 在该泄洪工况下, 当上游来流 $\mathrm{CO}_{2}$ 和 $\mathrm{CH}_{4}$ 的浓度分别高达 32.1 和 $0.145 \mathrm{mmol} / \mathrm{m}^{3}$ 时, 坝下近坝区的 $\mathrm{CO}_{2}$ 和 $\mathrm{CH}_{4}$ 的排放速率分别可高达 4032.3 和 $17.3 \mathrm{mmol} / \mathrm{s}$. 对于瀑布沟电站,在该泄洪工况下, 当上游来流 $\mathrm{CO}_{2}$ 和 $\mathrm{CH}_{4}$ 的浓度分别高达 32.1 和 0.145 $\mathrm{mmol} / \mathrm{m}^{3}$ 时, 坝下近坝区的 $\mathrm{CO}_{2}$ 和 $\mathrm{CH}_{4}$ 的释放可分别高达 2157.0 和 $1.65 \mathrm{mmol} / \mathrm{s}$. 对比可知, 对于坝面溢流或 空中挑流, 当温室气体人流浓度越高, 其坝下的温室气体排放速率也越高. 通过前述的分析可知, 上游来流 的温室气体浓度大小为影响坝下温室气体浓度分布的主要因素,进而影响了坝下温室气体排放速率分布. 

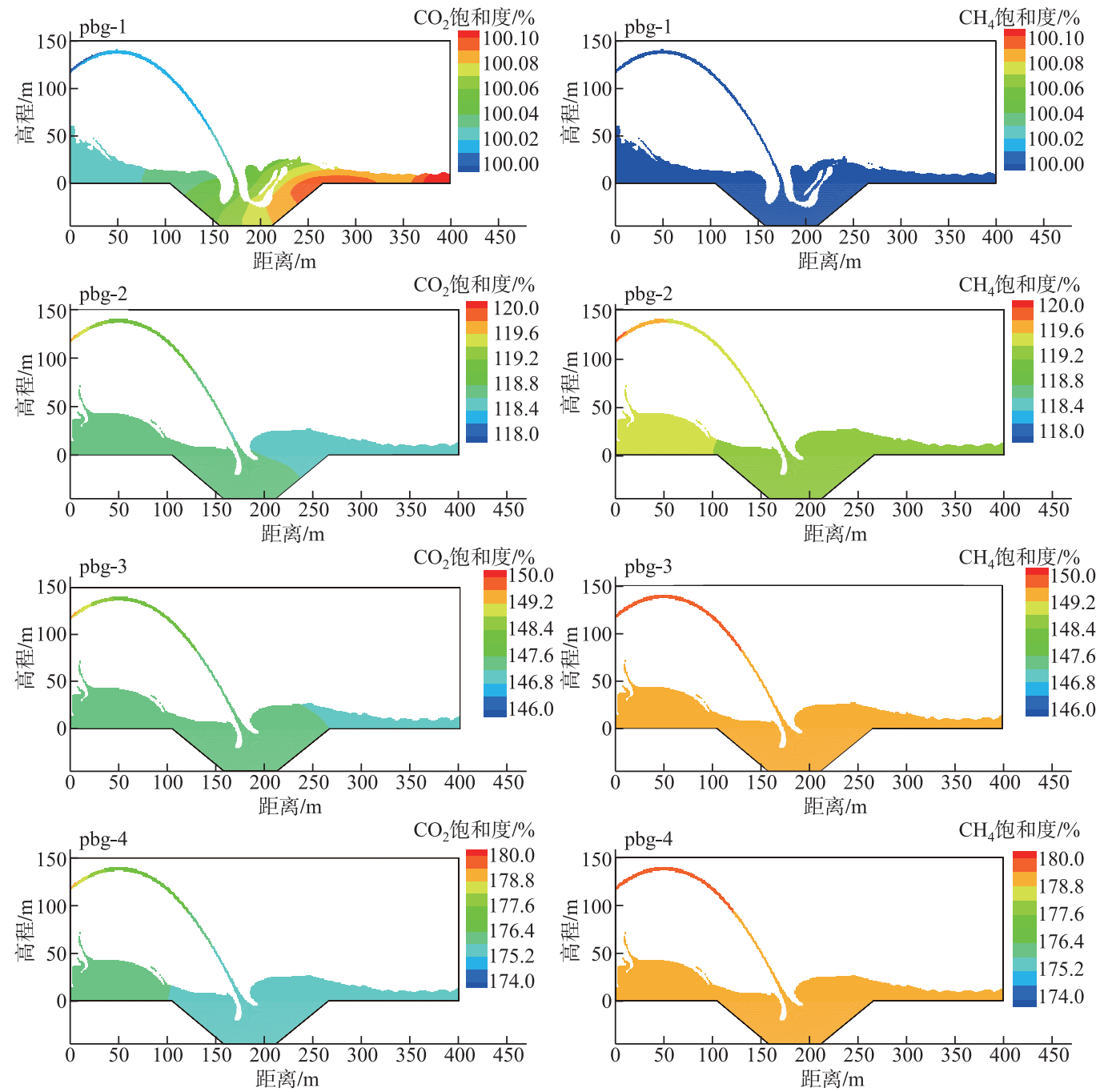

图 10 瀑布沟电站各工况温室气体过坝下泄模拟结果

Fig.10 The simulated results of GHG distribution with discharge under different working conditions of Pubugou Dam cases
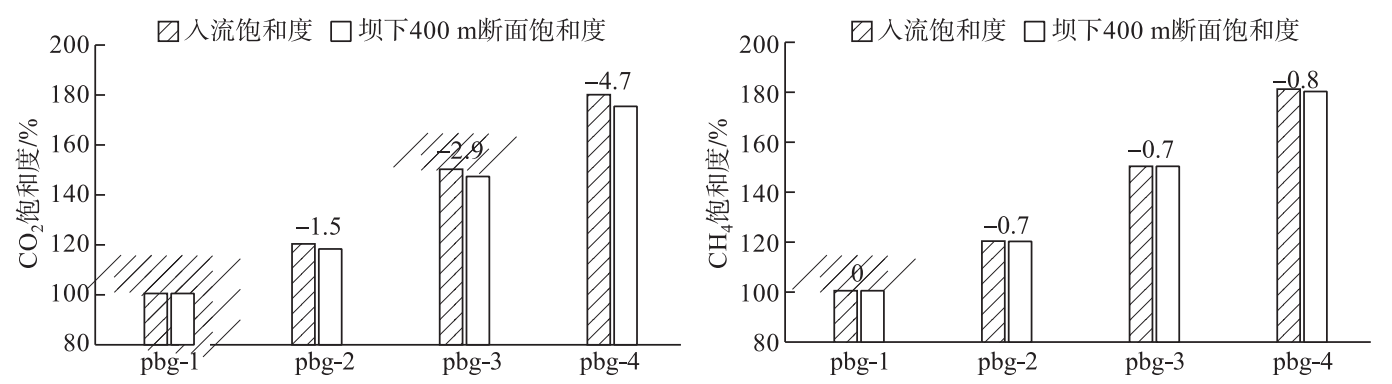

图 11 瀑布沟电站各工况温室气体消力池出口处浓度结果 (图中数字为饱和度差值)

Fig.11 The simulated results of GHG concentration at basin outlet under different working conditions of Pubugou Dam cases 
然而, 对于温室气体过坝下泄排放速率计算模型的研究目前还存在诸多不足. 水流过坝下泄的水动力 学模型涉及到高水头、大流速的水气两相流, 水动力学的模拟是探究过坝下泄中温室气体浓度及排放速率 的基础. 其中, 掺气浓度的计算结果直接影响到溶解气体的气泡量进而影响气液传质大小, 未来的研究将更 一步完善掺气浓度的计算模型, 为温室气体浓度的计算提供基础. 此外,已有众多学者对于库区自由液面传 质系数进行了研究 ${ }^{[18-20]}$, 而本研究中采用的库区自由液面传质计算公式, 针对高流速下的水流过坝下泄过 程,计算结果可能存在误差,未来将进一步完善高流速、强紊动条件下的温室气体自由液面传质系数计算. 在总溶解气体研究中, 是将溶解于水体中的气体按照空气组分比例进行分析, 而温室气体计算模型中, 只考 虑单一气体. 因此在模型计算中, 在气泡传质源项中采用空气组分比例来表示某种单一温室气体的气泡占 比, 而可能会与高压条件下实际空气溶解情况不符, 为计算带来误差. 未来的工作还将着重考虑在不同泄洪 流量、泄流流速等相关水力学因素的影响,并探索可以有效减缓温室气体排放的运行调度措施.

\section{4 结论}

通过引人过饱和总溶解气体的过坝下泄数值模拟模型, 分别考虑 $\mathrm{CO}_{2}$ 和 $\mathrm{CH}_{4}$ 两种温室气体的理化性质, 建立了温室气体过坝下排放速率计算模型. 模型基于 VOF 气液两相流模型, 采用 RANS 紊流模型, 分别考虑 了溶解气体与水体之间的气泡界面传质与自由液面传质. 通过对典型坝面溢流 (铜街子电站) 和空中挑流 (瀑布沟电站) 两种泄流方式下的温室气体浓度分布及坝下排放速率大小的模拟分析,认为由于大气中的温 室气体含量比例较低, 坝下发生的气液传质量受到限制, 因此上游来流的温室气体浓度大小为影响坝下温 室气体浓度分布的主要因素,并进而影响坝下温室气体排放速率的大小. 本研究为更进一步准确评估温室 气体释放通量提供新的研究思路和技术基础.

\section{5 参考文献}

[ 1 ] Solomon S, Qin D, Mannin M. Historical overview of climate change science. Intergovernmental Panel on Climate Change 2007. Cambridge: Cambridge University Press, 2007: 95-127.

[ 2 ] Crowley TJ. Causes of climate change over the past 1000 years. Science, 2000, 289(5477) : 270-277. DOI: 10.1126/science.289.5477.270

[ 3 ] Tett SFB, Stott PA, Allen MR et al. Causes of twentieth-century temperature change near the Earth's surface. Nature, 1999, 399(6736) : 569-572. DOI: 10.1038/21164.

[ 4 ] Tremblay A, Lambert M, Demers C. Introduction. Greenhouse gas emissions-Fluxes and processes. Berlin, Heidelberg: Springer, 2005: 21-34. DOI: 10.1007/978-3-540-26643-3_2.

[ 5 ] Sun ZY, Chen YB, Li C et al. Research of reservoir greenhouse gas emissions in China(2009-2019): Review and outlook. Journal of Hydraulic Engineering, 2020, 51(3): 253-267. [孙志禹, 陈永柏, 李羽等. 中国水库温室气体研究 (2009-2019) : 回顾与展望. 水利学报, 2020, 51(3) : 253-267.]

[ 6 ] Roehm C, Tremblay A. Role of turbines in the carbon dioxide emissions from two boreal reservoirs, Québec, Canada. Journal of Geophysical Research: Atmospheres, 2006, 111(D24) : D24102. DOI: 10.1029/2006JD007292.

[ 7 ] Abril G, Guérin F, Richard S et al. Carbon dioxide and methane emissions and the carbon budget of a 10-year old tropical reservoir (Petit Saut, French Guiana). Global Biogeochemical Cycles, 2005, 19 (4): GB4007. DOI: 10. 1029/2005GB002457.

[ 8 ] Soumis N, Duchemin É, Canuel R et al. Greenhouse gas emissions from reservoirs of the western United States. Global Biogeochemical Cycles, 2004, 18(3): GB3022. DOI: 10.1029/2003GB002197.

[ 9 ] Teodoru CR, Bastien J, Bonneville MC et al. The net carbon footprint of a newly created boreal hydroelectric reservoir. Global Biogeochemical Cycles, 2012, 26(2): GB2016. DOI: 10.1029/2011GB004187.

[10] Chanudet V, Descloux S, Harby A et al. Gross $\mathrm{CO}_{2}$ and $\mathrm{CH}_{4}$ emissions from the Nam Ngum and Nam Leuk sub-tropical reservoirs in Lao PDR. Science of the Total Environment, 2011, 409(24) : 5382-5391. DOI: 10.1016/j.scitotenv.2011. 09.018 .

[11] Huang JP, Lu JY, Li R et al. Numerical study on the cumulative effect of supersaturated TDG through the spillway. Ecohydrology \& Hydrobiology, 2021, 21(2) : 292-298. DOI: 10.1016/j.ecohyd.2021.01.003. 
[12] Wang YS, Politano M, Weber L. Spillway jet regime and total dissolved gas prediction with a multiphase flow model. Journal of Hydraulic Research, 2019, 57 (1) : 26-38. DOI: 10.1080/00221686.2018.1428231.

[13] Cheng XJ, Chen YC, Chen XW. Numerical simulation of dissolved oxygen concentration in the downstream of Three Georges Dam. Journal of Hydrodynamics: Ser A, 2009, 24(6) : 761-767. [ 程香菊, 陈永灿, 陈雪巍. 三峡工程坝身泄流下 游水体溶解氧浓度数值模拟. 水动力学研究与进展: A 辑, 2009, 24(6): 761-767.]

[14] Yang HX, Li R, Liang RF et al. A parameter analysis of a two-phase flow model for supersaturated total dissolved gas downstream spillways. Journal of Hydrodynamics: Ser B, 2016, 28 ( 4 ) : 648-657. DOI: 10. 1016/S1001-6058 ( 16 ) 60669-8

[15] Cheng XJ, Xie J, Yu DG et al. Calculated analysis of oxygen transfer from air bubble-water interface and turbulent water surface in microporous aeration systems. Transactions of the Chinese Society of Agricultural Engineering, 2013, 29(13): 190-199. [程香菊, 谢骏, 余德光等. 曝气增氧微气泡-水界面和水体表面的氧传质的计算分析. 农业工程学报, 2013, 29(13) : 190-199.]

[16] Cheng XJ, Xie YN, Zhu DT et al. Modeling re-oxygenation performance of fine-bubble-diffusing aeration system in aquaculture ponds. Aquaculture International, 2019, 27 (5) : 1353-1368. DOI: 10.1007/s10499-019-00390-6.

[17] Jähne B, Libner P, Fischer R et al. Investigating the transfer processes across the free aqueous viscous boundary layer by the controlled flux method. Tellus B , 1989, 41B(2) : 177-195. DOI: 10.1111/j.1600-0889.1989.tb00135.x.

[18] Cole JJ, Caraco NF. Atmospheric exchange of carbon dioxide in a low-wind oligotrophic lake measured by the addition of $\mathrm{SF}_{6}$. Limnology and Oceanography, 1998, 43(4) : 647-656. DOI: 10.4319/lo.1998.43.4.0647.

[19] MacIntyre S, Wanninkof R, Chanton JP. Trace gas exchange across the air-water interface in freshwater and coastal marine environments // Matson PA, Harriss RC eds. Biogenic trace gases: Measuring emissions from soil and water. New York: Blackwell Science, 1995: 52-97.

[20] Crusius J, Wanninkhof R. Gas transfer velocities measured at low wind speed over a lake. Limnology and Oceanography, 2003, 48(3) : 1010-1017. DOI: 10.4319/lo.2003.48.3.1010. 